\title{
An Evaluation of Service Quality in Higher Education: Marmara and Niğde Omer Halisdemir Universities' Department of Education Students
}

\author{
Sefer Ada ${ }^{1}$, Z. Nurdan Baysal ${ }^{2}$, Senem Seda Şahenk Erkan ${ }^{3, *}$ \\ ${ }^{1}$ Physical Education Department, Gedik University, Istanbul, Turkey \\ ${ }^{2}$ Primary Education Department, Marmara University, Istanbul, Turkey \\ ${ }^{3}$ Foreign Languages Department, Marmara University, Istanbul, Turkey
}

Copyright $\mathrm{C} 2017$ by authors, all rights reserved. Authors agree that this article remains permanently open access under the terms of the Creative Commons Attribution License 4.0 International License

\begin{abstract}
The purpose of this research is to evaluate the quality service in higher education in Marmara and Niğde Omer Halisdemir Universities' department of education students. This study was prepared using a screening model from quantitative research methods. The sample of this research comprised 886 university students attending the higher education institutions mentioned. The Scale of Service Quality in Higher Education Institutions is composed a data collection tool consisting of 28 items and 6 factors. The data of this research were collected during the 2016-2017 academic year. Gender, grade, university, and academic success were utilized in this study as personal variables. In the results of this study, the considerations of girls were higher than males regarding the academic position and institutional image. In addition, the perceptions of $3^{\text {rd }}$ grade students were higher than those of $4^{\text {th }}$ grade students according to academic position, institutional image, offered diploma programs, and physical opportunities. On the other hand, the academic success of university students was increased regarding all factors and total scores.
\end{abstract}

\section{Keywords Service Quality, Higher Education, Education Faculty}

\section{Introduction}

Globalization and technological developments today affect Turkey as well as all countries of the world. Adapting to such rapid changes require concentrated efforts. In order to adapt to all of the current global changes, the universities are in the first place to evoke change. Quality universities are the converters of the nations in exchange.

The concept of quality comes from a Latin word qualitas. Different definitions of this concept have been formed. The quality can be defined briefly as "compliance with standards". Furthermore, quality includes the elements of a product or service that will create a sense of satisfaction by meeting the basic needs of the target group with the different features it contains [1]. According to [2], "quality signifies also the conformity to the requirements of a product." It is possible to state that these requirements or standards meet the criteria for quality assurance if they are considered to be expressed as standards in the previous definition.

On the other hand, according to [3], quality is defined also as "a set of properties that include the ability of a good or service to deal with a particular need". The expression of the need for this definition also indicates requirements/standards or certain criteria. Also, quality determines how one might "create, design, produce, and provide after-sales services of the most economical, most usable, and always satisfying quality products" [4].

The importance of service quality in higher education has been gradually realized [5], and the role of service quality in higher education has attracted increased attention in the last two decades [6]. Higher education institutions must determine their needs and demands, as students are stakeholders and customers in this setting, and customer satisfaction is attached to service quality. Figure 1 shows the key stakeholder categories in higher education institutions as follows:

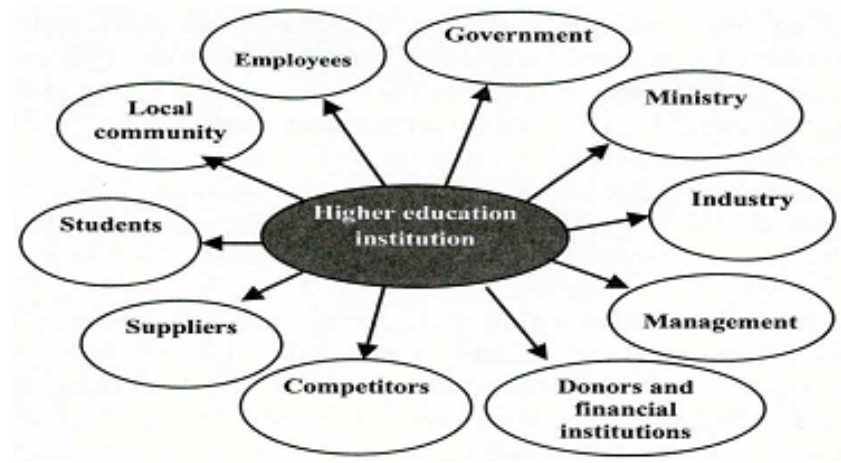

Figure 1. Higher education institution key stakeholder categories 


\section{Reference: [7]}

As indicated in Figure 1, the key stakeholder categories in higher education institution include people and organizations such as the employees, government, ministry, industry, management, donors and financial institutions, competitors, suppliers, and students within the local community.

The development of higher education service quality was attached to the organization's ability to ensure an overall climate and culture for change through its various decision-making and operating systems and human resource activities [8]. In higher education literature, [9] and [10] determined that students' perceived service quality is an antecedent to student satisfaction. In addition, the positive perceptions of service quality can provide student satisfaction, and satisfied students may then attract new students by engaging in positive word-of-mouth communication with acquaintances and friends, and they may return to the university to take other courses and other training programs [11]; [12]; [13]; [14]. Students' satisfaction has a positive influence on fundraising and student motivation [15].

While the subject of this research is higher education institutions, the service recipients are the customers, which are the students. The academic staff and other employees may also be considered service recipients. The quality elements in higher education are organized into six subcategories [12]:

(1) Concrete elements,

(2) Qualification,

(3) Attitude,

(4) Content,

(5) Presentation, and

(6) Reliability.

The concrete elements include equipment and facilities, modern equipment and facilities to facilitate transportation, good environmental support services (such as housing, sports, social services etc.), and adequate academic staff. The staff members demonstrate qualifications such as communication skills. Attitude shows also the individual ability to understand the needs of students, willingness to help, availability for help, and counseling. The content brings to the programs the students' future work, validity, computer use, communication skills, and teamwork. Effective presentation must involve consistency, impartiality of exams, feedback from students, and encouragement of students. Reliability is the capacity to stop talking, evaluate complaints, and solve problems [16]. Quality in higher education can be determined with five criteria: technique, process, infrastructure, interaction, and atmosphere [17]. Taking all these factors into consideration, it is understood that many factors must be present in order to be qualified to offer successful customer service in higher education.

In the $21^{\text {st }}$ century, the demand for university services has increased in every respect. A growing number of students are seeking education in good universities. Along with this increase in demand, the universities are producing more information and spreading the information through global opportunities. Therefore, as it is throughout the world, anxiety regarding quality in higher education is rising rapidly in Turkey. The number of students has rapidly increased with the rise in the number of universities and quotas opened in Turkey since the 1990s. However, the number of faculty members did not increase in parallel with the number of students, and the inadequacy of resources such as libraries and laboratories were brought to the agenda [18]. As with every institution, higher education institutions should also try to prove their quality in today's competitive world, where each innovation is rapidly spread and internalized, thus increasing the qualifications of such institutions. It is important that these institutions not only try to increase their qualifications on their own but also obtain the approval of commissions established for this purpose.

Quality evaluation in higher education in Turkey is being carried out by the Higher Education Academic Evaluation and Quality Improvement Commission, which is located in the Council of Universities. The aim of the commission in this regulation is to determine the strategies, processes, procedures, and principles related to the evaluation of academic and administrative services of higher education institutions; ensure the development of qualifications; and obtain the approval and recognition of quality levels in line with the strategic plan and goals of the Higher Education Council, and to update them when necessary and to inform higher education institutions that are situated in [19]. In this sense, it is noteworthy that established universities, institute quality, pass quality evaluations, and become branded in the world. These universities realize their quality improvement by taking into account what their future needs may be.

Under this concept, universities today are referred to as "Third-Generation Universities". These universities have a multi-functional structure to carry out research tasks as well as provide classes. If education is long term and quite expensive and errors are made, it seems natural to consider a number of factors in order to capture quality when the results are expected to take a long time to be realized. To address the required tasks, Turkey participated in a higher education quality project called the European Higher Education Area [20].

Since 2001, the training programs for "Leonardo," "Socrates," and the "Bologna Declaration," which were launched in the European Union, were organized in Turkey. Under this concept, the curriculums of the faculties in the universities were reorganized and were undergoing plans to be improved. This project sought to establish diplomas and teaching periods, with lifelong learning and common credits that can be applied all over the world. Thus, the harmonization of the European Credit Transfer System (ECTS) of all countries is supported. The ECTS aims to create field competencies and national qualifications ([20]. All these initiatives are evidence of their quest for quality.

It is important for universities to determine how much they 
can respond to the needs of the modern world and how their services are evaluated by the service providers. Therefore, this study may provide useful information that should be taken into consideration for the university administrations that conduct the research. For these reasons, this study was structured to determine how the students of Marmara and Niğde Omer Halisdemir Universities' education faculties evaluate their quality of service and take into consideration their existing situation, consider improving or increasing their quality of services, and determine the future strategies according to their students.

\section{Method}

The qualitative method and screening model were used in this research in order to investigate the perceptions of service quality in higher education in Marmara and Niğde Omer Halisdemir Universities' education students according to their gender, grade level, university, and success in classes. The screening model can be defined as research that is usually conducted on larger samples than the other studies are, while determining the participants' opinions or interests, skills, abilities, attitudes, etc., about a topic or event [21]

\subsection{Data Collection Tools}

In this study, the Personal Information Form was used to determine the gender, grade level, university, and course success levels of the students participating in the research. In addition, the Scale of Service Quality in Higher Education Institutions by Bektaş \& Akman (2013) was utilized for reliability and reliability calculations. In this study, the data were collected during the 2016-2017 academic year. This scale constituted of 28 items and a 5-item Likert-type scale. According to the validity studies of the scale, the $\mathrm{BMD} / \mathrm{KMO}$ value is 0.893 . The Cronbach Alpha values of the sub-factors and the reliability analysis are as follows [22]:

(1) First Factor: Institutional Administrative direction (10 items-Cronbach Alpha: 0.852);

(2) Second Factor: Institutional academic direction (6 items-Cronbach Alpha: 0.712);

(3) Third Factor: Institutional image (3 items-Cronbach Alpha: 0.870);

(4) Fourth Factor; Accessibility (3 items-Cronbach Alpha: 0.762);

(5) Fifth Factor: Diploma programs offered by institution (3 items-Cronbach Alpha: 0.717) and

(6) Sixth Factor: Institutional physical facilities (3 items-Cronbach Alpha: 0.761).

\subsection{Scope and Sample}

The sample of this study constitutes 886 students studying at Marmara and Niğde Omer Halisdemir Universities in the education faculties of the primary, maternity, social studies, mathematics, and science teaching departments. The appropriate sampling method was used when determining the sample of this study. With this type of sampling, the researcher created a sample with the most accessible responders to best achieve a representative group and maximize savings [23].

Table 1 shows the information about the sample of the study.

Table 1. Frequency and percentage of the distribution of students

\begin{tabular}{|c|c|c|c|c|c|c|c|c|}
\hline & f & $\%$ & & f & $\%$ & & f & $\%$ \\
\hline Scope & & & Scope & & & Scope & & \\
\hline Gender & & & University & & & Grade & & \\
\hline Female & 742 & 75.6 & Marmara University & 496 & 50.5 & $3^{\text {rd }}$ grade & 521 & 53.1 \\
\hline Male & 240 & 24.4 & Niğde Omer Halisdemir University & 394 & 49.5 & $4^{\text {th }}$ grade & 461 & 46.9 \\
\hline Total & 886 & 100.0 & Total & 886 & 100.0 & Total & 886 & 100.0 \\
\hline Department & f & $\%$ & Success Level & $\mathbf{f}$ & $\%$ & & & \\
\hline Maternity instructor & 96 & 29.5 & Weak & 30 & 3.1 & & & \\
\hline Primary instructor & 290 & 19.6 & Medium & 697 & 71.0 & & & \\
\hline Social Sciences Education & 194 & 19.8 & Good & 255 & 26.0 & & & \\
\hline Science Education & 156 & 15.9 & Total & 886 & 100.0 & & & \\
\hline Mathematics Education & 150 & 15.3 & & & & & & \\
\hline Total & 886 & 100.0 & & & & & & \\
\hline
\end{tabular}


According to Table 1, in the scope group, 742 students were female, 240 students were male, 496 were studying at Marmara University, and 394 students were trained at Niğde Omer Halisdemir University students. 521 students were educated for the $3^{\text {rd }}$ grade level, and 461 were trained for the $4^{\text {th }}$ grade level. 96 students in the department were maternity instructors, 290 students were in the department of primary instruction, 194 students in the department of Social Sciences Education, 156 were students in the department of science education, and 150 students were educated in the department of mathematics education. Finally, it was determined that 30 students had weak academic progress, 697 had medium success levels, and only 255 students had good results regarding their academic success.

\section{Findings}

The findings of this research were showed below.

T-test analysis findings about service quality in higher education related to the gender variables

The t-test analysis findings about service quality in higher education related to the genders variables are shown in Table 2 .

Table 2. T-test analysis results of service quality in higher education related to the gender variables

\begin{tabular}{|c|c|c|c|c|c|c|c|}
\hline & Gender & $\mathbf{N}$ & Mean & ss & $\mathbf{t}$ & df & Sig. \\
\hline \multirow{2}{*}{ Total } & Female & 672 & 3.0271 & .53338 & \multirow{2}{*}{2.732} & \multirow{2}{*}{884} & \multirow{2}{*}{.006} \\
\hline & Male & 214 & 2.9112 & .56200 & & & \\
\hline \multirow{2}{*}{$\begin{array}{c}\text { Factor } 1 \\
\text { Institutional administrative direction }\end{array}$} & Female & 672 & 3.0568 & 69519 & \multirow{2}{*}{3.05} & \multirow{2}{*}{884} & \multirow{2}{*}{$.002 *$} \\
\hline & Male & 214 & 2.8869 & .74941 & & & \\
\hline \multirow{2}{*}{$\begin{array}{c}\text { Factor } 2 \\
\text { Institutional academic direction }\end{array}$} & Female & 672 & 3.6699 & .65155 & \multirow{2}{*}{3.605} & \multirow{2}{*}{884} & \multirow{2}{*}{.000 * } \\
\hline & Male & 214 & 3.4836 & .67839 & & & \\
\hline \multirow{2}{*}{$\begin{array}{c}\text { Factor } 3 \\
\text { Institutional image }\end{array}$} & Female & 672 & 2.6503 & 1.21367 & \multirow{2}{*}{1.156} & \multirow{2}{*}{884} & \multirow{2}{*}{.248} \\
\hline & Male & 214 & 2.5421 & 1.12590 & & & \\
\hline \multirow{2}{*}{$\begin{array}{c}\text { Factor } 4 \\
\text { Accessibility }\end{array}$} & Female & 672 & 2.9578 & .87392 & \multirow{2}{*}{1.792} & \multirow{2}{*}{884} & \multirow{2}{*}{.074} \\
\hline & Male & 214 & 2.8318 & .96347 & & & \\
\hline \multirow{2}{*}{$\begin{array}{c}\text { Factor } 5 \\
\text { Diploma programs offered by } \\
\text { institution }\end{array}$} & Female & 672 & 2.7455 & .90145 & \multirow{2}{*}{-1.063} & \multirow{2}{*}{884} & \multirow{2}{*}{.310} \\
\hline & Male & 214 & 2.8224 & .98271 & & & \\
\hline \multirow{2}{*}{$\begin{array}{c}\text { Factor } 6 \\
\text { Institutional physical facilities }\end{array}$} & Female & 672 & 2.3700 & .88948 & \multirow{2}{*}{-.203} & \multirow{2}{*}{884} & \multirow{2}{*}{.851} \\
\hline & Male & 214 & 2,3847 & 1.02700 & & & \\
\hline
\end{tabular}

$* \mathrm{p}<.05$

As indicated in Table 2, when the students' perceptions of service quality are examined in terms of the differentiation related to their genders, it is determined that, among the six sub factors, the perceptions of girls are higher than the boys in only two factors (Factor 2: Institutional academic direction and Factor 3: Institutional Image).

T-test analysis findings about students' perceptions in service quality in higher education related to the grade variables

The t-test analysis findings about students' perceptions of service quality in higher education related to the grades variables were gathered in Table 3.

Table 3 presents the students' perceptions of service quality in higher education differentially or not related to the grades. The $3^{\text {rd }}$ grade students had higher perceptions than the $4^{\text {th }}$ grade students regarding factors such as institutional administrative direction (Factor 1), institutional academic direction (Factor 2), institutional image (Factor 3), diploma offered by the institution (Factor 5), and institutional physical possibilities (Factor 6). The $3^{\text {rd }}$ grade perceptions were also higher in total.

T-test analysis findings about students' perceptions of service quality in higher education related to the universities variables

The t-test analysis findings regarding students' perceptions of service quality in higher education related to the university variables are shown in Table 4. 
Table 3. T-test analysis results of students' perceptions of service quality in higher education related to the grade variables

\begin{tabular}{|c|c|c|c|c|c|c|c|}
\hline & Grade Level & $\mathbf{N}$ & Mean & ss & t & df & Sig. \\
\hline \multirow{2}{*}{$\begin{array}{l}\text { Factors } \\
\text { Total }\end{array}$} & $3^{\text {rd }}$ grade & 400 & 3.0813 & .52131 & \multirow{2}{*}{5.043} & \multirow{2}{*}{884} & \multirow{2}{*}{$.000 *$} \\
\hline & $4^{\text {th }}$ grade & 486 & 2.8992 & .55129 & & & \\
\hline \multirow{2}{*}{$\begin{array}{c}\text { Factor 1 } \\
\text { Institutional administrative direction }\end{array}$} & $3^{\text {rd }}$ grade & 400 & 3.1887 & .70510 & \multirow{2}{*}{8.264} & \multirow{2}{*}{884} & \multirow{2}{*}{$.000 *$} \\
\hline & $4^{\text {th }}$ grade & 486 & 2.8058 & .66283 & & & \\
\hline \multirow{2}{*}{$\begin{array}{c}\text { Factor } 2 \\
\text { Institutional academic direction }\end{array}$} & $3^{\text {rd }}$ grade & 400 & 3.6951 & .61047 & \multirow{2}{*}{3.499} & \multirow{2}{*}{884} & \multirow{2}{*}{$.000 *$} \\
\hline & $4^{\text {th }}$ grade & 486 & 3.5396 & .71233 & & & \\
\hline \multirow{2}{*}{$\begin{array}{c}\text { Factor } 3 \\
\text { Institutional image }\end{array}$} & 3rd grade & 400 & 2.4883 & 1.24215 & \multirow{2}{*}{-3.762} & \multirow{2}{*}{884} & \multirow{2}{*}{$.000 *$} \\
\hline & 4th grade & 486 & 2.7892 & 1.11057 & & & \\
\hline \multirow{2}{*}{$\begin{array}{c}\text { Factor } 4 \\
\text { Accessibility }\end{array}$} & $3^{\text {rd }}$ grade & 400 & 2.9925 & .82414 & \multirow{2}{*}{2.385} & \multirow{2}{*}{884} & \multirow{2}{*}{.017} \\
\hline & $4^{\text {th }}$ grade & 486 & 2.8483 & .97436 & & & \\
\hline \multirow{2}{*}{$\begin{array}{c}\text { Factor } 5 \\
\begin{array}{c}\text { Diploma programs offered by the } \\
\text { institution }\end{array} \\
\end{array}$} & $3^{\text {rd }}$ grade & 400 & 2.8471 & .96546 & \multirow{2}{*}{2.965} & \multirow{2}{*}{884} & \multirow[b]{2}{*}{$.003 *$} \\
\hline & $4^{\text {th }}$ grade & 486 & 2.6633 & .85611 & & & \\
\hline \multirow{2}{*}{$\begin{array}{c}\text { Factor } 6 \\
\text { Institutional physical facilities }\end{array}$} & $3^{\text {rd }}$ grade & 400 & 2.4122 & .92264 & \multirow{2}{*}{1.372} & \multirow{2}{*}{884} & \multirow{2}{*}{$.000 *$} \\
\hline & $4^{\text {th }}$ grade & 486 & 2.3267 & .92459 & & & \\
\hline
\end{tabular}

$* \mathrm{p}<.05$

Table 4. T-test analysis results of service quality in higher education related to the universities variables

\begin{tabular}{|c|c|c|c|c|c|c|c|}
\hline & University & $\mathbf{N}$ & Mean & ss & $\mathbf{t}$ & df & Sig. \\
\hline \multirow{2}{*}{$\begin{array}{c}\text { Factors } \\
\text { Total }\end{array}$} & Marmara University & 496 & 2.9950 & .51950 & \multirow[b]{2}{*}{-.257} & \multirow[b]{2}{*}{884} & \multirow[b]{2}{*}{.797} \\
\hline & $\begin{array}{c}\text { Niğde Omer Halisdemir } \\
\text { University }\end{array}$ & 390 & 3.0044 & .57080 & & & \\
\hline \multirow{2}{*}{$\begin{array}{c}\text { Factor } 1 \\
\text { Institutional administrative } \\
\text { direction }\end{array}$} & Marmara University & 496 & 3.0063 & .67953 & \multirow{2}{*}{-.450} & \multirow{2}{*}{884} & \multirow[b]{2}{*}{.657} \\
\hline & $\begin{array}{c}\text { Niğde Omer Halisdemir } \\
\text { University }\end{array}$ & 390 & 3.0279 & .75190 & & & \\
\hline \multirow{2}{*}{$\begin{array}{c}\text { Factor } 2 \\
\text { Institutional academic } \\
\text { direction }\end{array}$} & Marmara University & 496 & 3.6428 & .63462 & \multirow[b]{2}{*}{.907} & \multirow[b]{2}{*}{884} & \multirow[b]{2}{*}{.370} \\
\hline & $\begin{array}{l}\text { Niğde Omer Halisdemir } \\
\text { University }\end{array}$ & 390 & 3.6021 & .69665 & & & \\
\hline \multirow{2}{*}{$\begin{array}{c}\text { Factor } 3 \\
\text { Institutional } \\
\text { image }\end{array}$} & Marmara University & 496 & 2.5477 & 1.19725 & \multirow[b]{2}{*}{-2.155} & \multirow[b]{2}{*}{884} & \multirow[b]{2}{*}{.031} \\
\hline & $\begin{array}{c}\text { Niğde Omer Halisdemir } \\
\text { University }\end{array}$ & 390 & 2.7214 & 1.18274 & & & \\
\hline \multirow{2}{*}{$\begin{array}{c}\text { Factor } 4 \\
\text { Accessibility }\end{array}$} & Marmara & 496 & 2.8757 & .90714 & \multirow{2}{*}{-1.937} & \multirow{2}{*}{884} & \multirow{2}{*}{.053} \\
\hline & Niğde Omer Halisdemir & 390 & 2.9932 & .88173 & & & \\
\hline \multirow{2}{*}{$\begin{array}{c}\text { Factor } 5 \\
\begin{array}{c}\text { Diploma programs offered by } \\
\text { the institution }\end{array}\end{array}$} & Marmara University & 496 & 2.7379 & .89284 & \multirow[b]{2}{*}{-.954} & \multirow[b]{2}{*}{884} & \multirow[b]{2}{*}{.340} \\
\hline & $\begin{array}{l}\text { Niğde Omer Halisdemir } \\
\text { University }\end{array}$ & 390 & 2.7974 & .95739 & & & \\
\hline \multirow{2}{*}{$\begin{array}{c}\text { Factor } 6 \\
\text { Institutional physical facilities }\end{array}$} & Marmara University & 496 & 2.4852 & .95617 & \multirow[b]{2}{*}{4.091} & \multirow[b]{2}{*}{884} & \multirow[b]{2}{*}{$.000 *$} \\
\hline & $\begin{array}{l}\text { Niğde Omer Halisdemir } \\
\text { University }\end{array}$ & 390 & 2.2316 & .86188 & & & \\
\hline
\end{tabular}

*p $<.05$

As shown in Table 4, the students' perceptions of service quality in higher education are determined differentially or not related to the universities. In only the sixth sub-factor (Factor 6: Institutional physical facilities) the students' perceptions of service quality in higher education institution trained in Marmara University were higher than those of Niğde Omer Halisdemir University students.

ANOVA and Scheffe analysis findings about service quality in higher education related to the success levels variables

The ANOVA and Scheffe analysis findings about service quality in higher education related to the success levels variables are provided in Table 5. 
Table 5. ANOVA analysis findings about service quality in higher education related to the success levels variables

\begin{tabular}{|c|c|c|c|c|c|c|}
\hline Success Levels & Source of variance & $\begin{array}{l}\text { Sum of } \\
\text { squares }\end{array}$ & sd & $\begin{array}{l}\text { Mean } \\
\text { Square }\end{array}$ & $\mathbf{F}$ & Sig. \\
\hline \multirow{3}{*}{$\begin{array}{c}\text { Factor 1 } \\
\text { Institutional administrative direction }\end{array}$} & $\begin{array}{l}\text { Between } \\
\text { Groups }\end{array}$ & 6.781 & 2 & 3.391 & \multirow{3}{*}{6.776} & \multirow{3}{*}{$.001 *$} \\
\hline & $\begin{array}{l}\text { Within } \\
\text { Groups }\end{array}$ & 441.817 & 883 & \multirow[t]{2}{*}{.500} & & \\
\hline & Total & 448.599 & 885 & & & \\
\hline \multirow{3}{*}{$\begin{array}{c}\text { Factor } 2 \\
\text { Institutional academic direction }\end{array}$} & Between Groups & 3.877 & 2 & 1.939 & \multirow{3}{*}{4.451} & \multirow{3}{*}{$.012 *$} \\
\hline & Within Groups & 384.633 & 883 & \multirow[t]{2}{*}{.436} & & \\
\hline & Total & 388.510 & 885 & & & \\
\hline \multirow{3}{*}{$\begin{array}{c}\text { Factor } 3 \\
\text { Institutional } \\
\quad \text { image }\end{array}$} & $\begin{array}{l}\text { Between } \\
\text { Groups }\end{array}$ & 37.895 & 2 & 18.948 & \multirow{3}{*}{13.687} & \multirow{3}{*}{$.000 *$} \\
\hline & Within Groups & 1222.392 & 883 & \multirow[t]{2}{*}{1.384} & & \\
\hline & Total & 1260.288 & 885 & & & \\
\hline \multirow{3}{*}{$\begin{array}{c}\text { Factor } 4 \\
\text { Accessibility }\end{array}$} & $\begin{array}{c}\text { Between } \\
\text { Groups }\end{array}$ & 10.435 & 2 & 5.217 & \multirow{3}{*}{6.559} & \multirow{3}{*}{$.001 *$} \\
\hline & Within Groups & 702.338 & 883 & \multirow[t]{2}{*}{.795} & & \\
\hline & Total & 712.773 & 885 & & & \\
\hline \multirow{3}{*}{$\begin{array}{c}\text { Factor } 5 \\
\text { Diploma programs offered by the } \\
\text { institution }\end{array}$} & Between Groups & 37.181 & 2 & 18.590 & \multirow{3}{*}{22.967} & \multirow{3}{*}{$.000^{*}$} \\
\hline & Within Groups & 714.740 & 883 & \multirow{2}{*}{.809} & & \\
\hline & Total & 751.921 & 885 & & & \\
\hline \multirow{3}{*}{$\begin{array}{c}\text { Factor } 6 \\
\text { Institutional physical facilities }\end{array}$} & Between Groups & 18.158 & 2 & 9.079 & \multirow{3}{*}{10.871} & \multirow{3}{*}{$.000 *$} \\
\hline & Within Groups & 737.407 & 883 & \multirow{2}{*}{.835} & & \\
\hline & Total & 755.564 & 885 & & & \\
\hline \multirow{3}{*}{$\begin{array}{l}\text { Factors } \\
\text { Total }\end{array}$} & $\begin{array}{l}\text { Between } \\
\text { Groups }\end{array}$ & 5.421 & 2 & 2.710 & \multirow{3}{*}{9.388} & \multirow{3}{*}{$.000 *$} \\
\hline & Within Groups & 254.933 & 883 & \multirow[t]{2}{*}{.289} & & \\
\hline & Total & 260.354 & 885 & & & \\
\hline
\end{tabular}

$* \mathrm{p}<0.05$

Table 5 shows that the perception of students in perceptions of service quality in higher education varies according to the success levels. The differentiation in success level represented in the Scheffe Analysis is shown in Table 6 below. 

Halisdemir Universities' Department of Education Students

Table 6. Scheffe analysis findings about service quality in higher education related to the success level variables

\begin{tabular}{|c|c|c|c|c|c|}
\hline Successes Levels & (I) Success level & (J) Success Level & Medium (I-J) & Std Error & $\mathbf{p}$ \\
\hline \multirow{6}{*}{$\begin{array}{c}\text { Factor 1 } \\
\text { Institutional administrative direction }\end{array}$} & \multirow{2}{*}{ Weak } & Medium & $-.45276^{*}$ & .13673 & $.004 *$ \\
\hline & & Good & $-.34802 *$ & .14091 & .048 \\
\hline & \multirow{2}{*}{ Medium } & Weak & $.45276^{*}$ & .13673 & $.004 *$ \\
\hline & & Good & .10474 & .05302 & .143 \\
\hline & \multirow{2}{*}{ Good } & Weak & $.34802 *$ & .14091 & .048 \\
\hline & & Medium & -.10474 & .05302 & .143 \\
\hline \multirow{6}{*}{$\begin{array}{c}\text { Factor } 2 \\
\text { Institutional academic direction }\end{array}$} & \multirow{2}{*}{ Weak } & Medium & $-.38056^{*}$ & .12758 & $.012 *$ \\
\hline & & Good & $-.36111^{*}$ & .13147 & .023 \\
\hline & \multirow{2}{*}{ Medium } & Weak & $.38056^{*}$ & .12758 & $.012 *$ \\
\hline & & Good & .01945 & .04947 & .926 \\
\hline & \multirow{2}{*}{ Good } & Weak & $.36111^{*}$ & .13147 & .023 \\
\hline & & Medium & -.01945 & .04947 & .926 \\
\hline \multirow{6}{*}{$\begin{array}{c}\text { Factor } 3 \\
\text { Institutional image }\end{array}$} & \multirow{2}{*}{ Weak } & Medium & -.34818 & .22743 & .310 \\
\hline & & Good & $-.77116^{*}$ & .23438 & $.005^{*}$ \\
\hline & \multirow{2}{*}{ Medium } & Weak & .34818 & .22743 & .310 \\
\hline & & Good & $-.42298^{*}$ & .08819 & $.000 *$ \\
\hline & \multirow{2}{*}{ Good } & Weak & $.77116^{*}$ & .23438 & $.005 *$ \\
\hline & & Medium & $.42298^{*}$ & .08819 & $.000 *$ \\
\hline \multirow{6}{*}{$\begin{array}{c}\text { Factor } 4 \\
\text { Accessibility }\end{array}$} & \multirow{2}{*}{ Weak } & Medium & $-.60286^{*}$ & .17239 & $.002 *$ \\
\hline & & Good & $-.63889 *$ & .17766 & $.002 *$ \\
\hline & \multirow{2}{*}{ Medium } & Weak & $.60286^{*}$ & .17239 & $.002 *$ \\
\hline & & Medium & -.03603 & .06685 & .865 \\
\hline & Good & Weak & $.63889 *$ & .17766 & $.002 *$ \\
\hline & Medium & $\begin{array}{c}\text { Medium } \\
\text { Weak } \\
\text { Good } \\
\text { Weak } \\
\text { Medium } \\
\end{array}$ & $\begin{array}{c}.03603 \\
-.63889 \\
.03685 \\
.42298^{*} \\
.03603 \\
\end{array}$ & $\begin{array}{l}.06685 \\
.17766 \\
.06732 \\
.08819 \\
.06685 \\
\end{array}$ & $\begin{array}{l}.865 \\
.002 * \\
.850 \\
.000 * \\
.865 \\
\end{array}$ \\
\hline \multirow{6}{*}{$\begin{array}{c}\text { Factor } 5 \\
\text { Diploma offered by the institution }\end{array}$} & \multirow{2}{*}{ Weak } & Medium & -.24375 & .17391 & .375 \\
\hline & & Good & $-.67725^{*}$ & .17922 & $.001 *$ \\
\hline & \multirow{2}{*}{ Medium } & Weak & .24375 & .17391 & .375 \\
\hline & & Good & $-.43350 *$ & .06744 & $.000 *$ \\
\hline & \multirow{2}{*}{ Good } & Weak & $.67725^{*}$ & .17922 & $.001 *$ \\
\hline & & Medium & $.43350^{*}$ & .06744 & $.000 *$ \\
\hline \multirow{6}{*}{$\begin{array}{c}\text { Factor } 6 \\
\text { Institutional physical possibilities }\end{array}$} & \multirow{2}{*}{ Weak } & Medium & $-.58219^{*}$ & .17665 & $.005 *$ \\
\hline & & Good & -.33201 & .18204 & .190 \\
\hline & Medium & Weak & $.58219^{*}$ & .17665 & $.005 *$ \\
\hline & Medium & Good & $.25018^{*}$ & .06850 & $.001 *$ \\
\hline & & Weak & .33201 & .18204 & .190 \\
\hline & Good & & $-.25018^{*}$ & .06850 & $.001 *$ \\
\hline & & Medium & $-.45276^{*}$ & .13673 & $.004 *$ \\
\hline Factors & Weak & Medium & $-.43364 *$ & .10386 & $.000 *$ \\
\hline Total & Weak & Good & $-.46088^{*}$ & .10704 & $.000 *$ \\
\hline & Medium & Weak & $.43364^{*}$ & .10386 & $.000 *$ \\
\hline & Medium & Good & -.02724 & .04028 & .796 \\
\hline Total & Cond & Weak & $.46088^{*}$ & .10704 & $.000 *$ \\
\hline & Good & Medium & .02724 & .04028 & .796 \\
\hline
\end{tabular}


Table 6 shows that, in the first sub-factor, (institutional administrative direction), there was a significant difference in the level of $p<.05$ between the students with a weak success level and those with medium and good success levels. In addition, in this first factors, a significant difference was found in the $p<.05$ level in favor of the students with a medium success level between the medium- and weak-level students. In addition, in this first factor, there was a significant difference in the level of $p<.05$ in favor of the good and weak success-level students.

Examination of Table 6 reveals that, in the second sub factor (institutional academic direction), there was a significant difference in the level of $p<.05$ between students with a weak success level and students with medium and good success levels. In addition, in this first factor, a significant difference was determined at the $p<.05$ level in favor of the students with a medium success level between the medium and the weak level students. Furthermore, regarding this first factor, there was a significant difference in the level of $\mathrm{p}<.05$ in favor of students with good and weak success levels.

Table 6 shows that, in the third sub factor (institutional image), there was a significant difference in the level of $\mathrm{p}$ $<.05$ between students with a weak success level and students with medium and good success levels. In addition, regarding this first factor, a significant difference was found at the $\mathrm{p}<.05$ level in favor of the students with a medium success level between the students at medium and weak levels. For the first factor, there was a significant difference in the level of $\mathrm{p}<.05$ in favor of students with good and weak success levels.

In this scale cited above, in the fourth sub factor (accessibility), there was a significant difference in the level of $p<.05$ between students with a weak success level and students with medium and good success levels. In addition, in this first factor, a significant difference was determined in the $p<.05$ level in favor of the students with medium success levels between the students at medium and weak levels. For this first factor, there was a significant difference in the level of $p<.05$ in favor of good and weak success level students'.

In this scale, in the fifth sub factor (diploma offered by the institution), there was a significant difference in the level of $p$ $<.05$ between students with a weak success level and students with medium and good success levels. In addition, for this first factor, a significant difference was found in the $p$ $<.05$ level in favor of the students with a medium success level between the students with medium and weak levels. In addition, regarding the first factor, there was a significant difference in the level of $p<.05$ in favor of students with good and weak success levels.

In this scale, in the sixth sub factor (institutional physical possibilities), there was a significant difference in the level of $p<.05$ between students with a weak success level and students with medium and good success levels. In addition, in this first factor, a significant difference was revealed in the $\mathrm{p}<.05$ level in favor of the students with a medium success level between the students with medium and weak levels. Furthermore, in this first factor, there was a significant difference in the level of $p<.05$ in favor of students with good and weak success levels.

In the factors of this scale, there was a significant difference in the level of $p<.05$ between students with a weak success level and students with medium and good success levels. In addition, in this first factor, a significant difference was found in the $p<.05$ level in favor of the students with a medium success level between the students with medium and weak levels. Regarding this first factor, it was determined that there was a significant difference in the level of $p<.05$ in favor of students with good and weak success levels.

\section{Results}

The results of this research are summarized below:

(1) Among the six sub factors, in only two factors (Factor 2: Institutional academic direction and Factor 3: Institutional Image), the perceptions of girls regarding service quality in higher education were higher than the boys.

(2) The $3^{\text {rd }}$ grade students had higher-level perceptions than the $4^{\text {th }}$ grade students of service quality in higher education in some factors, such as institutional administrative direction (Factor 1), institutional academic direction (Factor 2), institutional image (Factor 3), diploma offered by the institution (Factor 5), and institutional physical possibilities (Factor 6).

(3) Among all six sub-factors, in only the sixth sub factor (Factor 6: Institutional physical facilities) were the students' perceptions of service quality in higher education institutions at Marmara University determined to be higher than those of Niğde Ömer Halisdemir University students.

(4) When the success levels of university students increase, their perceptions of service quality in higher education can improve directly in all sub factors and total factors of this scale.

The perception of service quality in higher education is higher in third grade than in fourth grade. Regarding this concept, it is seen that the perception of higher education service quality decreases among the students in the fourth grade. This result can be produced because of the program. When the programs are examined, it can be interpreted that the courses related to the profession take place in the third grade more, and it does not affect the perception of the third grade students. In this case, it can be argued that the students were in the final year due to their focus on KPSS.

Also, it can be determined that the students of Marmara University have a better chance of evaluating the physical possibilities of the university because they have access to the cultural richness of Istanbul, and the campus is in a central position. 
On the other hand, according to the results, when the success level of the university students increases, their perceptions of service quality in higher education rise. The students who had weak success levels perceived weak service quality in higher education in some sub factors, such as institutional administrative and academic directions, institutional image, accessibility, diploma offered by the institution, and also institutional physical possibilities. This is a thought-provoking result. It is possible to make an interpretation that the expectations of the students with weak achievement level are increasing.

\subsection{Discussions}

The students' perceptions of service quality in higher education institutions indicated a significant difference according to the year of the universities' establishment. Significant differences were observed in the sub factors of service quality perceptions, such as institutional image, diploma programs offered by the institution, and also institutional physical possibilities [21].

According to the results of the present study, female students in Marmara and Niğde Ömer Halisdemir Universities in the education faculty produced evaluations at a higher level than males according to the institutional academic direction and image in terms of service quality perception higher education. Similarly, in the study conducted by [24], the perceptions of female students were found to be higher regarding some sub factors than males. But, on the other hand, the perceived quality of service among the students who graduated from different higher education institutions receiving pedagogical formation in Necmettin Erbakan University by [21] did not show any significant difference according to gender.

The present study determined that all students trained in $3^{\text {rd }}$ grade in the related universities had a higher level of education than $4^{\text {th }}$ grade in terms of perceptions of service quality in higher education in five sub factors, not only in the fourth sub factor of this scale. Similarly, there is a significant difference in the grades of university students in perceptions of service quality in higher education in terms of temporal, conceptual, and structural sub factors of the study conducted by [24] in a similar way in the higher education service quality perceptions of students at Manchester University in the USA higher education institutions compared to their grade levels. In another work of research by [25], there is a significant difference in their grades of university students in terms of perceptions of service quality perceptions in higher education in terms of implementation processes, expectations, and physical possibilities according to the grade variable of the students in Matej Bel and Belgrade Universities. Furthermore, in the study conducted by [26] in the Çanakkale 18 March University were found significant differences between students' grades and their perceptions of service quality in higher education. In this framework, the findings of the studies mentioned in our study represent similar results in the grade level variable of the students differentiating in perceptions in perceptions of service quality in higher education with the different sub factors explained above.

In the present study, Marmara University has received a better evaluation than Niğde Ömer Halisdemir University in terms of the physical possibilities of the institution for better service quality. Similarly, [27] examined North African universities and determined that the perceptions of service quality in higher education changed according to the universities in terms of physical possibilities.

The significant difference was determined in all sub factors in perceptions of service quality in higher education according to the success levels of the students.

In the studies by [28] and [24] within the scope of international research, the perceptions of service quality in higher education showed significant differences regarding many sub factors in higher education according to their students' success levels. At the same time, in the study conducted by [26], the significant differences were found between the success levels of the students and the perceptions of service quality in higher education in some sub factors of higher education institutions.

\subsection{Recommendations}

In conclusion, the suggestions are expressed below:

(1) One of the most important goals of our university's undergraduate program for teaching education should be to improve the perceptions of service quality in higher education.

(2) Maximum improvements may be proposed to improve the existing conditions in the universities. Measures may be increased according to the satisfactions of male students, especially in terms of academic direction and image of education.

(3) Precautions should be taken to ensure that the academic background/level of success of employees have the knowledge and experience that can provide and support the expectations of the weakest students.

(4) The universe may include higher education institutions in a specific province or region instead of two higher education institutions.

(5) The research method can be organized in quantitative, qualitative, and also mixed methods.

(6) More detailed information on each sub factor in perceptions of service quality in higher education can be obtained through qualitative research methods.

\section{REFERENCES}

[1] Şimşek, M. (2000). Sorularla toplam kalite yOnetimi ve kalite güvence sistemleri (Quality administration with questions and quality assurance systems). İstanbul: Alfa Publications. 
[2] Crosby, P. B. (1979). Quality is free. USA: New York: McGraw-Hill, Advanced Engineering Study.

[3] American Council on Education. (2015). International higher education partnerships: A global review of standards and practices. USA: Washington, DC: Author.

[4] Ishikawa, P. B. (1985). What is total quality control. USA: Washington, Prentice Hall.

[5] Tan, W. \& Simpson, K. (2008). Overseas educational experience of Chinese students: an evaluation of service quality experience in New Zealand. Journal of Research in International Education, 7(1), 93- 112.

[6] Jelena, L. (2010). Determinants of service quality in higher education. Interdisciplinary Management Research, 6, 631-647.

[7] Đonlogič, S. \& Fazlič, S. (2015). Quality assessment in higher education using the SERVQUAL model. Management, 20 (1), $39-57$.

[8] Mosadeghard, A. M. (2006). A Survey total quality in Iran: Barriers to successful implementation of total quality management. The Q\&M Magazine, 8 (6), 606-625.

[9] Browne, B., Kaldenberg, D., Browne, W. \& Brown, D. (1998). Student as customers: factors affecting satisfaction and assessments of institutional quality. Journal of Marketing for Higher Education, 8 (3), 1-14.

[10] Guolla, M. (1999). Assessing the teaching quality to student satisfaction relationship: applied customer satisfaction research in the classroom. Journal of Marketing Theory and Practice, 7 (3), 87- 97.

[11] Marzo-Navarro, M., Pedraja-Iglesias, M. \& Rivera-Torres, M.P. (2005). Measuring customer satisfaction in summer courses. Quality Assurance in Education, 13 (1), 53-65.

[12] Wiers-Jenssen, J., Stensaker, B. \& Grogaard, J.B. (2002). Student satisfaction: towards an empirical deconstruction of the concept. Quality in Higher Education, 8 (2), 183-95.

[13] Mavondo, F.T., Tsarenko, Y. \& Gabbott, M. (2004). International and local student satisfaction: resources and capabilities perspective. Journal of Marketing for Higher Education, 14 (1), 41-60.

[14] Schertzer, C.B. \& Schertzer, S.M.B. (2004). Student satisfaction and retention: A conceptual model. Journal of Marketing for Higher Education, 14 (1), 79-91.

[15] Elliott, K.M. \& Shin, D. (2002). Student satisfaction: an alternative approach to assessing this important concept. Journal of Higher Education Policy and Management, 24 (2), 197-209.

[16] [Ali, M. \& Shastri, R. K. (2010). Implementation of total quality management in higher education. Asian Journal of Business Management, 2(1), 9-16.

[17] Zineldin, M., Akdağ, H. C. \& Vasicheva, V. (2011). Assessing quality in higher education: New criteria for evaluating students' satisfaction. Quality in Higher Education, 17 (2), 231-243.

[18] Kurnaz, O. (2010). YüksekOğretimde araștırma kalitesinin incelenmesi (Investigation of research quality in higher education). Doctoral Dissertation, İstanbul: Marmara University Education Sciences Institute.

[19] CoHE (2007). Türkiye'nin yüksekOğretim stratejisi (Higher education strategy in Turkey). Turkey: Ankara: CoHE.

[20] Büyüköztürk, Ş., Çakmak, E. K., Akgün, O. E., Karadeniz, Ş. \& Demirel, F. (2008). Bilimsel araştırma yöntemleri (Scientific research methods) ( $2^{\text {nd }}$ Edition). Ankara: PegemA Publication.

[21] Yavuz, M. \& Gülmez, D. (2016). YüksekOğretimde hizmet kalitesi algısının değerlendirilmesi (The assessment of service quality perception in higher education). Journal of Education and Science, 41 (184), 251-265.

[22] Bektaş, H. \& Ulutürk Akman, S. (2013). Yükseköğretimde Hizmet Kalitesi Olçeği: Güvenilirlik ve geçerlilik analizi (Service Quality Scale in Higher Education: Reliability and validity analysis). Journal of Istanbul University Economical Faculty Econometry Istatistics, No: 18, 116-133.

[23] Güzel Şahin, G. (2011). Üniversite düzeyinde turizm eğitiminde hizmet kalitesi beklenti ve algısına yOnelik Ankara'da bir araştırma (A research on expectation and perception of service quality in Tourism Education at University level in Ankara). Journal of Management Researches, 3(4), 49-65.

[24] Beaumont, D. (2012). Service quality in higher education: the students' view point. BSc (Honors) in Management, USA: Manchester University Manchester Business School.

[25] Dado, J., Taborecka Petrovicova, J., Riznic, D. \& Rajic, T. (2011). An empirical investigation into the construct of higher education service quality. International Review of Management and Marketing, 1(3), 30-42.

[26] Yelkikalan, N., Sümer, B. \& Temel, S. (2006). Fakültelerin değerlendirmelerinde Oğrencilerin algıları: Biga İktisadi ve İdari Bilimler Fakültesi Oğrencileri üzerine bir araştırma (Evaluation of faculties about students' perceptions: A research in Biga Economical and Administrative Faculty students'). Journal of Selçuk University Karaman Economical and Administrative Sciences Faculty, No: 1, 144-160.

[27] Soni, S. S. (2015). Service quality, student satisfaction and brand equity: A case study of select South African universities. Doctoral Dissertation, Africa: Durban, KwaZulu-Natal University.

[28] Farahmandian, S., Minavand, H. ve Afshardost, M. (2013). Perceived service quality and student satisfaction in higher education. IOSR Journal of Business and Management (IOSR-JBM), e-ISSN: 2278-487X, p-ISSN: 2319-7668, 12(4), 65-74. 